\title{
Structural Changes of Ultrathin Cub- PrO2(111)/Si(111) Films Due to Thermally Induced Oxygen Desorption
}

Article in The Journal of Physical Chemistry C · January 2014

DOI: 10.1021/jp4082917

CITATIONS

4

8 authors, including:

\section{Sebastian Gevers}

Universität Osnabrück

19 PUBLICATIONS 117 CITATIONS

SEE PROFILE

\section{Marcus Bäumer}

Universität Bremen

256 PUBLICATIONS $\quad 7,414$ CITATIONS

SEE PROFILE
READS

24
Andreas Schaefer

Lund University

29 PUBLICATIONS 283 CITATIONS

SEE PROFILE

\section{H. Zoellner}

Institute for High Performance Microelectron... 25 PUBLICATIONS 88 CITATIONS

SEE PROFILE

Some of the authors of this publication are also working on these related projects: 


\section{Structural changes of ultra thin cub- $\mathrm{PrO}_{2}(111) / \mathrm{Si}(111)$ films due to thermally induced oxygen desorption}

H. Wilkens ${ }^{1}$, S. Gevers ${ }^{1}$, S. Röhe ${ }^{2}$, A. Schaefer ${ }^{2}$, M. Bäumer ${ }^{2}$, M. H. Zoellner ${ }^{3}$, T. Schroeder ${ }^{3,4}$ and J. Wollschläger ${ }^{a} 1$

${ }^{1}$ Fachbereich Physik, Universität Osnabrück, Barbarastr. 7, D-49069 Osnabrück, Germany

2 Institute of Applied and Physical Chemistry, University of Bremen, Leobener Str. NW2, D-28359 Bremen, Germany

3 IHP, Im Technologiepark 25, D-15236 Frankfurt(Oder),

Germany

${ }^{4}$ BTU Cottbus, Institute of Physics, Konrad-Zuse-Str.1, D-03046 Cottbus, Germany

(Dated: 13 December 2013)

The structural changes of ultra thin $\mathrm{PrO}_{2}(111)$ films grown on $\mathrm{Si}(111)$ due to thermal reduction in ultra high vacuum are investigated. Therefore, praseodymia films are treated with oxygen plasma to obtain completely oxidized $\mathrm{PrO}_{2}$ films. The reduction of these films is studied with thermal desorption spectroscopy combined with ex-situ x-ray diffraction measurements. It is shown that the distinct desorption peaks of molecular oxygen are correlated with stabilization of several intermediate phases of praseodymia. The most intense signal of desorbing oxygen is observed at $\approx 650$ K. It is coupled to the strongest impact on the crystalline structure since we observe the transition $\mathrm{PrO}_{2} \rightarrow \operatorname{Pr}_{5} \mathrm{O}_{9}$. Further oxygen desorption leads to additional phase transitions with laterally coexisting phases. Grazing incidence x-ray diffraction measurements reveal that the initial pinning to the lateral lattice constant of hexagonal $\mathrm{Pr}_{2} \mathrm{O}_{3}(0001)$ is not breached due to the plasma treatment. This hexagonal pinning is released during the oxygen desorption so that the crystalline structure of the praseodymia films does not only expand vertically but also laterally.

\footnotetext{
a) email: joachim.wollschlaeger@uos.de
} 


\section{INTRODUCTION}

Rare earth oxides (REOs) exhibit the potential to improve several applications in different fields ranging from heterogeneous catalysis ${ }^{1,2}$ to microelectronics ${ }^{3,4}$. For instance, on the one hand, it has been demonstrated that oxide films are suitable as buffer layers for semiconductors as, e.g., $\mathrm{Si}^{5}, \mathrm{Ge}^{6}, \mathrm{InP}^{7}$ or $\mathrm{GaN}^{8}$. On the other hand, the high oxygen mobility of REOs and their large oxygen storage capacity gain particular interest in the field of catalysis. ${ }^{9}$ Thus, promising applications of REOs are the three-way automotive exhaust catalysis ${ }^{10}, \mathrm{CO}$ oxidation $^{11}$ or oxidative dehydrogenation of ethane and the oxidative coupling of methane ${ }^{12,13}$.

The reactivity and selectivity of these reactions is strongly influenced by structural properties, since lattice oxygen directly participates in surface redox reactions based on the Mars-van-Krevelen mechanism. ${ }^{14}$ Here, praseodymia has attracted attention due to the mobility of lattice oxygen. ${ }^{9}$ The structure and, thus, the chemical properties of praseodymia can be tuned by preparation of intermediate phases ${ }^{15,16}$ or doping with other rare earth metals. ${ }^{17}$. Hence huge efforts were made to study redox properties of praseodymia powders close to equilibrium ${ }^{18}$ as well as the oxygen desorption kinetics of praseodymia powders. ${ }^{19}$

However, the understanding of the reaction mechanisms on an atomic scale is quite challenging and model catalysts are needed to obtain deeper insight in the physical and chemical properties of the catalytic processes. Thus, the aim of the research presented here was to prepare well defined systems which can be used as model catalysts. Thin film systems give a great opportunity as model catalysts to study the questions mentioned above since, compared to powder studies, they offer reduced complexity combined with high structural control. ${ }^{20-22}$ Before thin film systems can be used as model catalysts, however, it has to be proved whether these systems show similar or different redox properties compared to the corresponding bulk structures. For instance, the structure and, thus, the redox properties can be altered by substrate induced effects like the previously reported lateral pinning at the interfaces of ultra thin films. ${ }^{23}$ Therfore, we combine surface sensitive techniques with techniques to control the 'bulk' structure of the praseodymia film in our study on the thermally induced desorption kinetics of oxygen.

We focus on the relation between oxygen release and structural properties for ultrathin praseodymia films grown on $\mathrm{Si}(111)$ substrates since praseodymia is characterized by the 
highest degree of oxygen mobility of all REOs. ${ }^{9}$ These films are promising candidates for such model systems since epitaxial growth can be achieved resulting in films with high crystalline order. ${ }^{24-26}$ Different oxide phases ranging from hex- $\mathrm{Pr}_{2} \mathrm{O}_{3}(0001)$ and cub- $\mathrm{Pr}_{2} \mathrm{O}_{3}(111)$ to slightly reduced $\mathrm{PrO}_{2-\Delta}(111)$ can be reached by post deposition annealing in oxygen atmosphere at different pressures. ${ }^{23,27}$

Recently, we demonstrated that fully oxidized $\mathrm{PrO}_{2}(111)$ films with clean surfaces can be prepared by exposure to oxygen plasma. ${ }^{28,29}$ Therefore, we present a study on the thermal oxygen release from this highest oxidation state. On one hand, the oxygen release has been controlled by thermal programmed desorption (TPD) detecting molecular oxygen. On the other hand, the structural properties of the reduced praseodymia film has been studied by synchrotron radiation based x-ray diffraction (SR-XRD) to elucidate the role of the structural changes due to oxygen release at the surface.

\section{EXPERIMENT}

An ultra thin hexagonal $\mathrm{Pr}_{2} \mathrm{O}_{3}$ film with (0001) orientation is grown via MBE on a clean Boron doped $4^{\prime \prime} \mathrm{Si}(111)$ wafer $(\sigma=5-15 \Omega \mathrm{cm})$ as described in Refs. ${ }^{26,30,31}$. Afterwards, the film is ex-situ oxidized by annealing in 1 bar oxygen atmosphere at $970 \mathrm{~K}$ for $30 \mathrm{~min}$. This treatment leads to the formation of a (111) oriented epitaxial cubic $\mathrm{PrO}_{2-\Delta}$ film with fluorite structure having a small amount of oxygen vacancies. ${ }^{23}$ For these films, a thickness of $16.5 \mathrm{~nm}$ is determined by x-ray reflection (XRR) and crystalline quality monitored by X-ray diffraction (XRD) (data not shown).

The wafer is split into several pieces which are loaded into an ultra high vacuum (UHV) chamber (base pressure $10^{-10}$ mbar) equipped with a RF-plasma source and TPD system. ${ }^{32}$ Each sample is plasma treated to obtain a fully oxidized cub- $\mathrm{PrO}_{2}(111)$ film with a clean surface. $^{28,29}$ Subsequently, in-situ TPD measurements monitoring the mass of molecular oxygen are performed with an annealing rate of $1 \mathrm{~K} / \mathrm{s}$ as described in Ref. ${ }^{28}$ (accuracy of the temperature measurements is $\pm 10 \mathrm{~K}$ ). In addition, the desorption signals of atomic oxygen, carbon dioxide, carbon monoxide, and water are simultaneously monitored. For each sample, the molecular oxygen desorption is stopped after a characteristic desorption peak appeared in the spectrum and the sample is rapidly quenched to room temperature (RT) (cf. Fig 1). Thereafter, to determine the structure of the samples ex-situ XRD and grazing incidence 


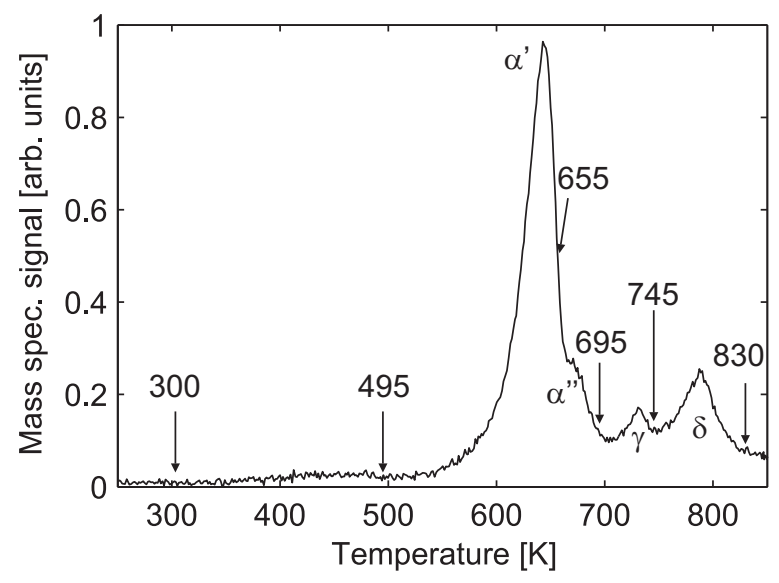

FIG. 1. TPD spectrum of molecular oxygen performed with an annealing rate of $1 \mathrm{~K} / \mathrm{s}$. For different samples the desorption is stopped after a certain characteristic peak is reached (arrows) and the sample is immediately cooled to RT to perform (GI)XRD experiments. The $\alpha^{\prime}, \gamma$ and $\delta$ peak are in good agreement with previous powder studies. ${ }^{19}$

(GI)XRD experiments are carried out at beamline BW2 at DESY (Hamburg, Germany) using a photon energy of $10 \mathrm{keV}$. The time span between sample preparation and (GI)XRD measurements is kept a small as possible, as well as, samples have been transported under nitrogen atmosphere to suppress any reaction with atmospheric oxygen.

\section{A. Oxygen-TPD}

The plasma treated samples show only desorption signals for molecular oxygen while the other monitored species are not observed. Hence, the surface is free of contaminations which was also shown by x-ray photoelectron spectroscopy (XPS) ${ }^{28}$ Fig.1 presents the TPD spectrum of molecular oxygen for a plasma treated sample. For the different sample the desorption is stopped after a characteristic peak is reached (arrows) and the samples are immediately cooled to RT to perform (GI)XRD experiments. Comparing the spectrum with the powder study of $\mathrm{PrO}_{2}$ done by Takasu et al. ${ }^{19}$, a quite similar overall behavior is observed. In accordance with the powder study three distinct peaks are visible which are labeled $\alpha^{\prime}(\approx 650 \mathrm{~K}), \gamma(\approx 730 \mathrm{~K})$ and $\delta(\approx 790 \mathrm{~K})$.

In contrast to the powder study our spectra show two additional features. Prior to the strongest desorption signal $\alpha^{\prime}$ a low intense, but broad peak at $\approx 450 \mathrm{~K}$ can be observed. This peak may be attributed to oxygen species which are weakly bound to the surface. Furthermore, the spectrum exhibits an additional weak peak at the high temperature wing 

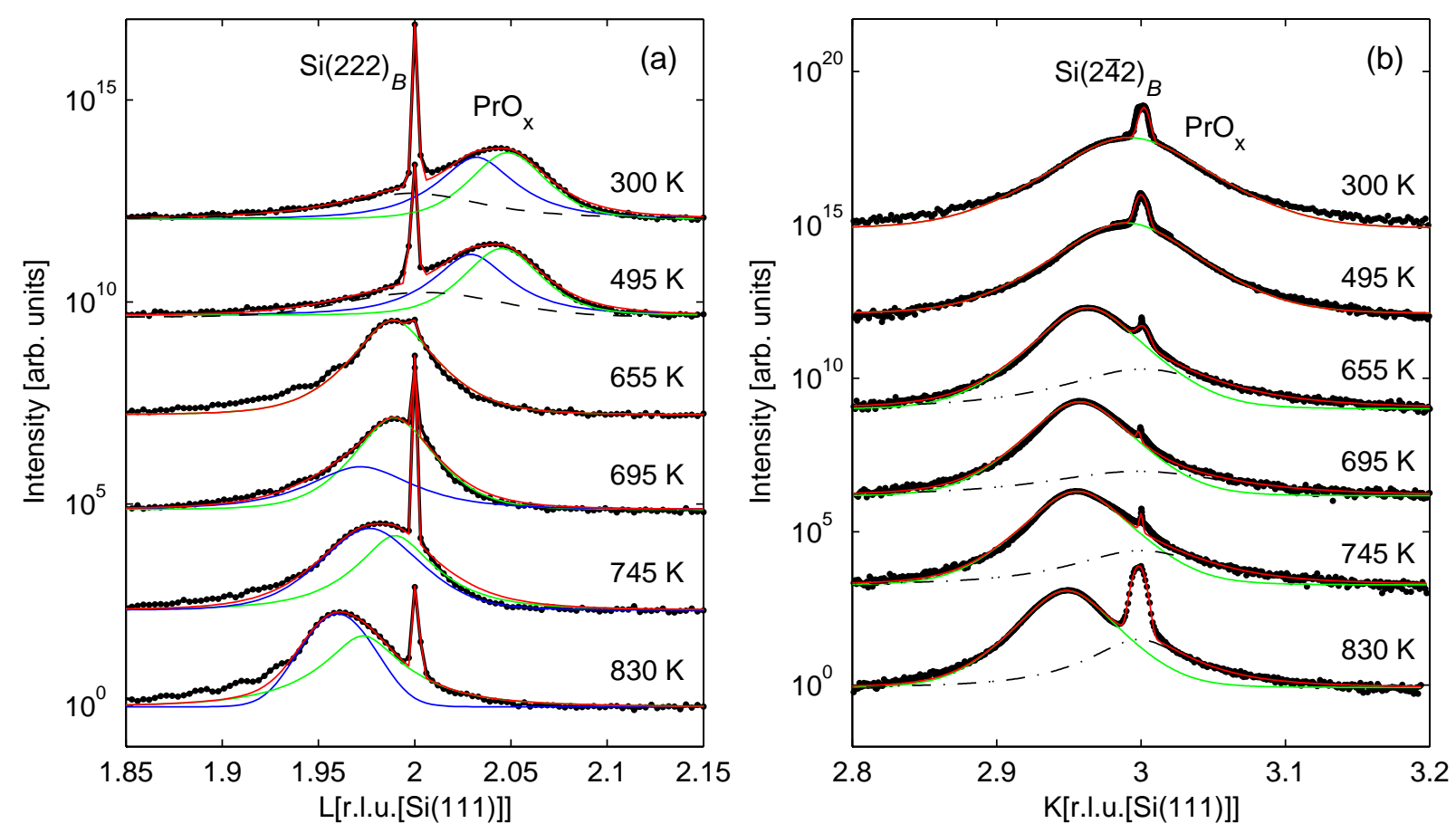

FIG. 2. (a) CTRs near the $(222)_{B}$ Si Bragg peak for the different quenched samples. The oxide peak is fitted with two Lorentzian functions representing the two major praseodymia phases. In addition, a broad peak (dashed lines ) is observed for the non-reduced films which can be attributed to a very thin $\operatorname{Pr}_{6} \mathrm{O}_{11}$ interface layer (see also Ref. ${ }^{29}$ ). (b) In-plane scans near the $(2 \overline{4} 2)_{B}$ Si Bragg peak. The oxide peak is described by a Lorentzian function. A weak second peak under the substrate peak is observed (dash-dotted line). We assume that this contribution is based on the interface roughness of the silicon substrate resulting in a broadening of the $(2 \overline{4} 2)_{B}$ Si Bragg peak.

of the $\alpha^{\prime}$ peak denoted by $\alpha^{\prime \prime}$.

\section{B. XRD}

Having reached a characteristic desorption peak, the samples are rapidly quenched to room temperature. The (GI)XRD measurements for the different quenched samples are shown in Fig. 2. The axes are labeled with reciprocal lattice units $(\mathrm{HKL})_{S}$ corresponding to the hexagonal $\mathrm{Si}(111)$ surface coordinate system, while the Bragg peaks are labeled with $(\mathrm{HKL})_{B}$ indices corresponding to the bulk structure. In Fig. 2 (a) the specular $\theta-2 \theta$ scans close to the kinematically forbidden $\operatorname{Si}(222)_{B}$ peak are analyzed: The broad diffracted intensity is given by the Bragg peak due to the praseodymia film and can be used to determine the vertical layer spacing of the oxide film. There is still a weak sharp $\operatorname{Si}(222)_{B}$ peak visible due to the non spherical electron density distribution. Therefore, the weak diffraction signal 
of the silicon bulk does not significantly interfere with the diffraction signal of the oxide film and the $\operatorname{Si}(222)_{B}$ peak can be used to calibrate the measurement.

Previous XRD investigations have shown by analysis within the kinematic diffraction theory that two laterally coexisting oxide phases are present in the praseodymia films which can be described by a two column model. ${ }^{31,33}$ We describe the oxide Bragg peaks by two Lorentzian functions representing the two coexisting praseodymia phases. For the nonreduced samples a third peak has to be used to describe the data. This is necessary since a minor reduced praseodymia phase $\left(\mathrm{Pr}_{6} \mathrm{O}_{11}\right)$ is pinned to the interface as described in detail in Ref. $^{29}$

Furthermore, in-plane scans near the $\operatorname{Si}(2 \overline{4} 2)_{B}$ Bragg peak are performed and the oxide peak is also fitted with a Lorentzian function (cf. Fig. 2 (b)) to determine the lateral layer spacing. A second weak broad in-plane peak beneath the sharp silicon Bragg peak is observed. We assume that this feature is based on the interface roughness between the $\mathrm{Si}$ substrate and the oxide film.

The vertical layer distance $c$ ([111] direction) and lateral layer distance $a$ ([1]10] direction) as determined from the peak positions are shown in Fig. 3. The amount of each species is calculated from the intensity of the Lorentzian functions and displayed as size of the circles around the markers. Bigger cycles represent major parts and small cycles minorities.

The two praseodymia species obtained for the plasma treated film exhibit a vertical layer distance of $3.06 \AA$ and $3.09 \AA$ which can be attributed to $\mathrm{PrO}_{2}$ and $\mathrm{PrO}_{2-\Delta}$, respectively. ${ }^{28,29}$ The corresponding lateral layer distance shows that the oxide film is pinned close to lateral layer distance of bulk hex- $\mathrm{Pr}_{2} \mathrm{O}_{3}$. Obviously, the $\mathrm{PrO}_{2}$ film is strongly distorted since the vertical layer distance is clearly smaller than its bulk value while the lateral layer distance is larger.

The first broad desorption peak at $\approx 450 \mathrm{~K}$ has no impact on the structure of the praseodymia films since the XRD data of the sample quenched from $495 \mathrm{~K}$ to RT does not show any significant changes in comparison with the plasma treated sample (cf. XRD data presented in Fig. 2).

On the contrary, the large amount of oxygen desorbing at $\approx 650 \mathrm{~K}$ has a strong impact on the structure of the praseodymia film. The film has homogeneous vertical structure since only one phase is needed to model the data. The determined vertical layer distance is in the region of $\operatorname{Pr}_{6} \mathrm{O}_{11}$ and fringes close to the oxide Bragg peak appear indicating an 

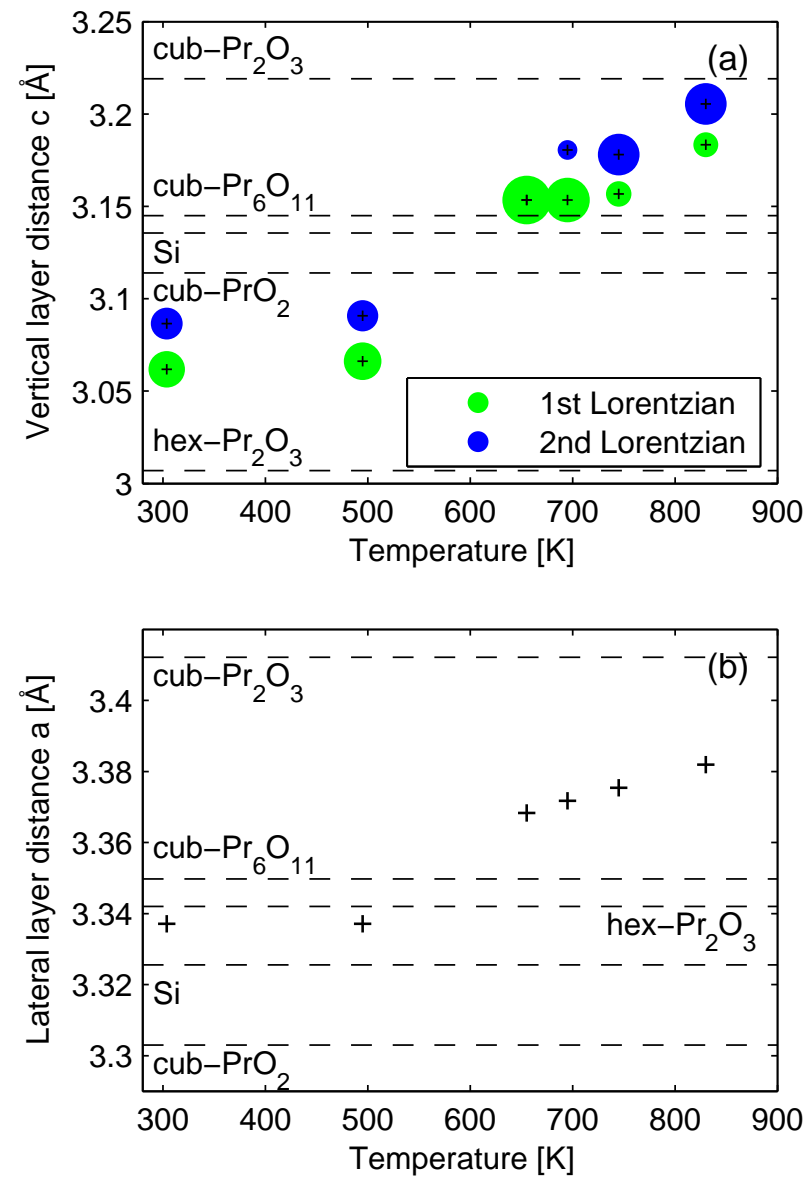

FIG. 3. (a) Vertical layer distance determined from the peak positions close to the $\mathrm{Si}(222)_{B}$ peak. The colored circles represent the intensity of the two functions. (b) Lateral layer distance obtained from the (GI)XRD measurements.

improvement of the film homogeneity. The lateral pinning to the hex- $\mathrm{Pr}_{2} \mathrm{O}_{3}$ layer distance, however, is breached due to the fact that the film has a lateral layer distance between $\operatorname{Pr}_{6} \mathrm{O}_{11}$ and cub- $\mathrm{Pr}_{2} \mathrm{O}_{3}$. Thus, the film has a different stoichiometry than $\operatorname{Pr}_{6} \mathrm{O}_{11}$.

For the following desorption peaks the previously visible fringes are diminished and the film is split into two lateral coexisting phases again. This points to roughening of the praseodymia films as well as of the interface (which is detected as diffuse diffraction signal close to the $\operatorname{Si}(2 \overline{4} 2)_{B}$ Bragg peak). In addition, the lateral layer distances shift to larger values. The sample quenched at $830 \mathrm{~K}$ shows again clear fringes close to the oxide Bragg peak indicating a reordering of the praseodymia layer and reduced roughness. Furthermore, the two praseodymia phases exhibit a vertical layer distance close to the value of cub- $\operatorname{Pr}_{2} \mathrm{O}_{3}$. On the contrary, the film has a lateral layer distance of $3.38 \AA$ which is far below the 


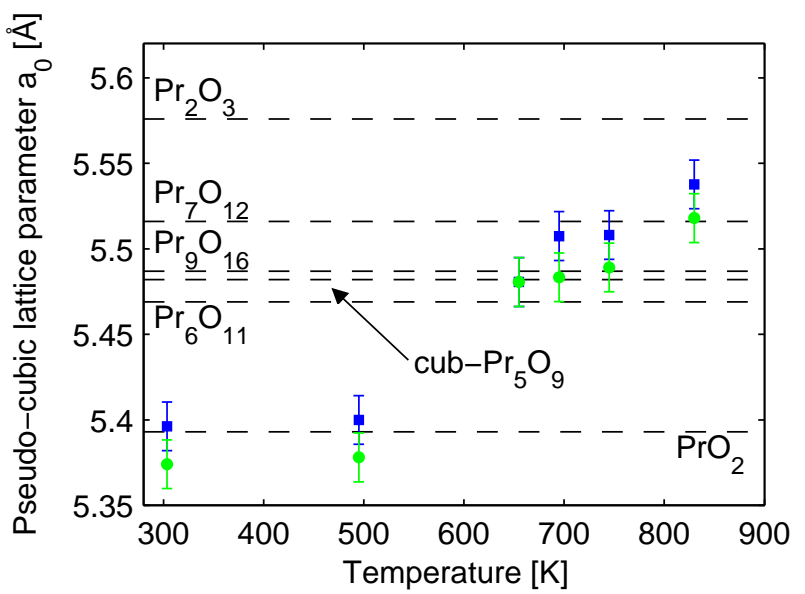

FIG. 4. Calculated pseudo-cubic lattice constants $a_{0}$ for the two columns. The literature values of the praseodymia phases stable at RT are represented by dashed lines.

value of cub- $\mathrm{Pr}_{2} \mathrm{O}_{3}$. Thus, the elastic distortion of the epitaxial praseodymia film has to be considered to clarify the phases gained due to reduction of the film.

The intermediate phases of praseodymia are all based on the same fluorite type $\mathrm{PrO}_{2}$ lattice with ordered oxygen vacancies. ${ }^{16,18}$ Therefore, they can be described by a pseudocubic lattice parameter $a_{0}$. Zaumseil et al. have shown that the cubic phases of praseodymia are tetragonally distorted by compressive strain if grown on $\mathrm{Si}(111)$ and a Poisson ratio of $\nu=0.32$ has been determined. ${ }^{35}$ Using this Poisson ratio we can recalculate the pseudo-cubic lattice parameter $a_{0}$ from our data obtained from the distorted film via

$$
a_{0}=\frac{\sqrt{3} c+A(2 \sqrt{2} a / \sqrt{3})}{1+A} \text { with } A=\frac{2 \nu}{1-\nu}
$$

The results are presented in Fig. 4. In the low temperature region only the $\mathrm{PrO}_{2}$ phase is present. The first desorption peak $\left(\alpha^{\prime}\right)$ results in a phase transition to the $\operatorname{Pr}_{6} \mathrm{O}_{11}-\operatorname{Pr}_{9} \mathrm{O}_{16}$ region. The following $\mathrm{a}_{0}$ values exhibit only small changes. The sample quenched at $830 \mathrm{~K}$ exhibits a smaller cubic lattice constant than expected for the cub- $\mathrm{Pr}_{2} \mathrm{O}_{3}$ phase.

\section{DISCUSSION}

The (GI)XRD data of the plasma treated sample clearly shows a lateral layer distance close to the value of hex- $\mathrm{Pr}_{2} \mathrm{O}_{3}$. This result implies that the in Ref. ${ }^{23}$ reported exclusive lateral hexagonal pinning during growth is not breached by the plasma treatment. Further- 
more, the oxygen release around a temperature of $450 \mathrm{~K}$ can not be related to any structural change. We assume that the plasma treatment results in additional weakly bound adsorbed oxygen at the surface which desorbs at low temperatures. These species are probably adsorbates due to the plasma treatment (e.g. molecular oxygen or recombining atomic oxygen).

Major structural changes can be attributed to the strongest desorption peak $\alpha^{\prime}$ at $\approx$ $650 \mathrm{~K}$. It is observed that the entire film exhibits only one praseodymia phase which is accompanied by an increase of crystalline order of the film. The breach of the lateral hexagonal pinning leads to a roughening of the substrate interface. The exact phase after the massive oxygen release is difficult to determine within experimental error since the pseudocubic lattice parameter of the $\operatorname{Pr}_{6} \mathrm{O}_{11}, \operatorname{Pr}_{5} \mathrm{O}_{9}$ and $\operatorname{Pr}_{9} \mathrm{O}_{16}$ species lie in a very narrow region. We observe the best agreement to the $\operatorname{Pr}_{5} \mathrm{O}_{9}$ phase which is stabilized under these conditions instead of the $\operatorname{Pr}_{6} \mathrm{O}_{11}$ phase which is the most stable under ambient conditions. ${ }^{16,34}$ This results coincides with the results of the powder study reported by Takasu et. al. ${ }^{19}$ They attribute the low stability of $\mathrm{Pr}_{6} \mathrm{O}_{11}$ under conditions of TPD to the lower activation energy of oxygen desorption of $\operatorname{Pr}_{6} \mathrm{O}_{11}$ compared to the other praseodymia phases. Hence, we assume that after the overcoming of the miscibility gap between $\operatorname{PrO}_{2}$ and $\operatorname{Pr}_{6} \mathrm{O}_{11}$, the film is directly further reduced to a $\mathrm{Pr}_{5} \mathrm{O}_{9}$ species which exhibits a higher activation energy for oxygen desorption.

Stopping the desorption process at $695 \mathrm{~K}$ after the shoulder $\alpha^{\prime \prime}$ of the $\alpha^{\prime}$ desorption peak appears in the desorption spectrum, the diffraction measurements show an additional splitting into two phases. A small amount of the $\mathrm{Pr}_{9} \mathrm{O}_{16}$ species is formed while the majority of the film still exhibits the $\operatorname{Pr}_{5} \mathrm{O}_{9}$ stoichiometry.

Having increased the temperature ramp to $745 \mathrm{~K}$ the major part of the film exhibits a large amount of the $\mathrm{Pr}_{7} \mathrm{O}_{12}$ phase while a small amount of to the $\operatorname{Pr}_{9} \mathrm{O}_{16}$ species is still present. Hence, we assume that the $\gamma$ desorption signal is caused by the transition $\operatorname{Pr}_{9} \mathrm{O}_{16}$ $\rightarrow \mathrm{Pr}_{7} \mathrm{O}_{12}$.

The sample quenched after the last desorption peak $\delta$ exhibits small remains of the $\operatorname{Pr}_{7} \mathrm{O}_{12}$ phase while the majority of the film consists of a non-stoichiometric phase. Based on Vegards law the stoichiometry of $\mathrm{PrO}_{1.64}$ is estimated via the pseudo-cubic lattice parameter. These result implies that the film is not fully reduced and further diffraction peaks are expected at higher temperatures. Thus, after the desorption process characterized by the $\delta$ TPD peak a non-stoichiometric but metastable phase is generated. Various of these phases are also 
reported for powder samples. ${ }^{36}$ Obviously the metastable phase $\mathrm{PrO}_{1.64}$ is quite stable since higher desorption temperatures are necessary to form completely reduced $\operatorname{Pr}_{2} \mathrm{O}_{3}$.

This results may also be an effect of the temperature ramped in our experiment by $1 \mathrm{~K} / \mathrm{s}$. In comparison, Gevers et al. ${ }^{33}$ showed that $\mathrm{PrO}_{2-\Delta}$ films can almost completely be reduced to $\mathrm{Pr}_{2} \mathrm{O}_{3}$ for isothermal desorption (ITD) performed for 30 min at $573 \mathrm{~K}$. Note, the samples investigated in this study are in contrast to our study not free of surface contamination which could influence the reduction behavior. The study also reports the lateral depinning of praseodymia films from the low temperature lateral structure close to hex- $\mathrm{Pr}_{2} \mathrm{O}_{3}$ to larger values.

In summary, quenching the samples after every characteristic desorption peak we mostly observe the lateral coexistence of two separate phases making it very difficult to form single phase praseodymia films. Obviously, the thermal budget to reduce the $\operatorname{PrO}_{x}(x=1.71-1.83)$ phases is very similar so that the transition from one phase to another can not be completed before a third phase starts to grow. This is a consequence of the narrow stoichiometric window of $\mathrm{PrO}_{x}(x=1.71$ - 1.83) exhibiting various phases with similar oxygen content.

\section{CONCLUSION}

The presented study correlates the structural changes of a thin praseodymia film with the release of molecular oxygen at the surface. The first process leading to the desorption peak $\alpha^{\prime}$ in the TPD spectrum has the strongest impact regarding the structural changes and the oxygen release at the surface. The previously reported exclusive lateral hexagonal pinning is clearly breached during this oxygen release. Furthermore, we attribute the different desorption peaks to the several phase transitions. This gives the opportunity to study distinct praseodymia phases which is a prerequisite for understanding and tuning the selectivity and activity of the model catalyst. Our detailed Bragg peak analysis showed that mostly not single phases are formed after characteristic desorption peaks are reached. Therefore, it has to be taken into account that similar to the powder studies it is very difficult to prepare single phase films. In future catalysis studies it has to be considered that in case of non-equilibrium preparation phases can only stabilized in coexistences. Thus, tuning of the catalytic reactions may be quite challenging. 


\section{ACKNOWLEDGMENT}

The authors thank the Deutsche Forschungsgemeinschaft (DFG) via grant no. WO533/161, BA1710/17-1 and SCHR1123/4-1 for financial support. Furthermore, D. Novikov is thanked for beamline support during synchrotron experiments carried out at HASYLAB/DESY.

\section{REFERENCES}

${ }^{1}$ Bhaskaran, S.K.; Bhat, V.T. Catalytic activity of $\mathrm{CeO}_{2}$ and $\operatorname{Pr}_{2} \underline{O}_{3} \underline{\text { for the liquid-phase }}$ benzylation of o-xylene to 3,4-dimethyldiphenylmethane. React. Kinet. Catal. Lett. 2002, 75, 239-243.

${ }^{2}$ Köhn, R. D.; Pan, Z.; Sun, J.; Liang, C. Ring-Opening Polymerization of D,L-Lactide with Bis(trimethyl triazacyclohexane)Praseodymium Triflate. Catal. Commun. 2003, 4, 33-37.

${ }^{3}$ Kwo, J. ; Hong, M.; Busch, B.; Muller, D. A.; Chabal, Y. J.; Kortan, A. R.; Mannaerts, J. P.; Yang, B.; Ye, P.; Gossmann, H.; Sergent, A. M.; Ng, K. K.; Bude, J.; Schulte, W. H.; Garfunkel, E.; Gustafsson, T. Advances in high k gate dielectrics for Si and III-V semiconductors. J. Cryst. Growth 2003, 251, 645-650.

${ }^{4}$ Bedell, S.W.; Reznicek, A.; Fogel, K.; Ott, J.; Sadana, D.K. Strain and lattice engineering for Ge FET devices. Mater. Sci. Semicond. Process. 2006, 9, 423-436.

${ }^{5}$ Seifarth, O.; Dietrich, B.; Zaumseil, P.; Giussani, A.; Storck, P.; Schroeder, T. Integration of strained and relaxed silicon thin films on silicon wafers via engineered oxide heterostructures: Experiment and theory. J. Appl. Phys. 2010, 108, 073526.

${ }^{6}$ Giussani, A.; Rodenbach, P.; Zaumseil, P.; Dabrowski, J.; Kurps, R.; Weidner, G.; Müssig, H.-J.; Storck, P.; Wollschläger, J.; Schroeder, T. Atomically smooth and single crystalline $\mathrm{Ge}(111) /$ cubic- $\mathrm{Pr}_{2} \mathrm{O}_{3}(111) / \mathrm{Si}(111)$ heterostructures: Structural and chemical composition study. J. Appl. Phys. 2009, 105, 033512.

${ }^{7}$ Saint-Girons, G.; Regreny, P.; Largeau, L.; Patriarche, G.; Hollinger, G. Monolithic integration of InP based heterostructures on silicon using crystalline $\mathrm{Gd}_{2} \underline{\mathrm{O}}_{3} \underline{\text { buffers. }}$ Appl . Phys. Lett. 2007, 91, 241912.

${ }^{8}$ Tarnawska, L.; Giussani, A.; Zaumseil, P.; Schubert, M.; Paszkiewicz, R.; Brandt, O.; 
Storck, P.; Schroeder, T. Single crystalline $\mathrm{Sc}_{2} \mathrm{O}_{3} / \mathrm{Y}_{2} \mathrm{O}_{3}$ heterostructures as novel engineered buffer approach for GaN ingregration on Si(111). J. Appl. Phys. 2010, 108, 063502.

${ }^{9}$ Antoshin, G. V.; Minachev, K. M.; Dmitriev, D. R. Mobility of oxygen and catalytic properties of rare earth oxides with respect to oxidation of hydrogen. Russ. Chem. Bull. 1967, 16, 1793-1795.

${ }^{10}$ Diwell, A. F.; Rajaram, R. R.; Shaw, H. A.; Treux, T. J. The role of ceria in three-way catalysts, in Catalysis Automotive Pollution Control, Vol. 71, Elsevier, Amsterdam, 1991.

11 Takasu, Y.; Matsui, M.; Matsuda, Y. The catalytic contribution of the lattice oxygen atoms of praseodymium oxide to the oxidation of carbon monoxide. J. Catal. 1982, 76, 61-64.

${ }^{12}$ Asami, K.; Kusakabe, K.-i.; Ashi, N.; Ohtsuka, Y. Synthesis of ethane and ethylene from methane and carbon dioxide over praseodymium oxide catalysts. Appl. Catal. A 1997, 156, 43-56.

${ }^{13}$ Kennedy, E. M.; Cant, N. W.; Comparison of the oxidative dehydrogenation of ethane and oxidative coupling of methane over rare earth oxides. Appl. Catal. 1991, 75, 321-330.

$\underline{14}$ Borchert, Y.; Sonström, P.; Wilhelm, M.; Borchert, H.; Bäumer, M. Nanostructured Praseodymium Oxide: Preparation, Structure, and Catalytic Properties. J. Phys. Chem. C 2008, 112, 3054-3063.

${ }^{15}$ Hyde, B. G.; Bevan, D. J. M.; Eyring, L., On the Praseodymium+Oxygen System. Philos. Trans. R. Soc. London. Ser. A1966, 259, 583-615.

${ }^{16}$ Adachi G.; Imanaka, N. The Binary Rare Earth Oxides. Chem. Rev.1998, 98, 1479-1514.

${ }^{17}$ Zhou, G.; Grote, J. Thermodynamic Investigation of the Redox Properties for CeriaHafnia, Ceria-Terbia, and Ceria-Praseodymia Solid Solutions. J. Phys. Chem. B 2008, 112, 9869-9875.

${ }^{18}$ Eyring, L.; Beanziger N.C. On the Structure and Related Properties of the Oxides of Praseodymium. J. Appl. Phys. 1962, 33, 428-433.

$\underline{19}$ Takasu, Y.; Matsui, M.; Tamura, H.; Kawamura, S.; Matsuda, Y.; Tojoshima, I. Temperature-Programmed Desorption on the Unstable Lattice Oxygen of Praseodymium Oxide. J. Catal. 1981, 69, 51-57.

${ }^{20}$ Campbell, T. Ultrathin metal films and particles on oxide surfaces: structural, electronic and chemisorptive properties. Surf. Sci. Rep. 1997, 27, 1-111.

${ }^{21}$ Henry, R. Surface studies of supported model catalysts. Surf. Sci. Rep. 1998, 31, 231-233, 
235-325.

${ }^{22}$ Bäumer M.; Freund, H.-J. Metal deposits on well-ordered oxide films. Prog. Surf. Sci. 1999, 61, 127-198.

${ }^{23}$ Weisemoeller, T.; Deiter, C.; Bertram, F.; Gevers, S.; Giussani, A.; Zaumseil, P.; Schroeder, T.; Wollschläger, J. Epitaxy of Single Crystalline $\mathrm{PrO}_{2}$ films on $\mathrm{Si}(111)$. Appl. Phys. Lett. 2008, 93, 032905.

${ }^{24}$ Tarsa, E. J.; Speck, J. S.; Robinson, McD. Pulsed laser deposition of epitaxial silicon/h$\underline{\mathrm{Pr}_{2}} \underline{\mathrm{O}}_{3} /$ silicon heterostructures. Appl. Phys. Lett. 1993, 63, 539-541.

${ }^{25}$ Liu, J. P.; Zaumseil, P.; Bugiel, E.; Osten, H. J. Epitaxial growth of $\operatorname{Pr}_{2} \mathrm{O}_{3}$ on $\mathrm{Si}(111)$ and the observation of a hexagonal to cubic phase transition during postgrowth $\mathrm{N}_{2}$ annealing. Appl. Phys. Lett. 2001, 79, 671-673.

${ }^{26}$ Schaefer, A.; Schroeder, T.; Lupina, G.; Borchert, Y.; Dabrowski, J.; Wenger, Ch.; Bäumer, M. Heteroepitaxial praseodymium sesquioxide films on $\operatorname{Si}(111)$ : A new model catalyst system for praseodymium oxide based catalysts. Surf. Sci. 2007, 601, 1473-1480.

${ }^{27}$ Schroeder, T.; Zaumseil, P.; Weidner, G.; Wenger, Ch.; Dabrowski, J.; Müssig, H.-J.; Storck, P. On the epitaxy of twin-free cubic (111) praseodymium sesquioxide films on Si(111) J. Appl. Phys. 2006, 99, 014101.

${ }^{28}$ Schaefer, A.; Gevers, S.; Zielasek, V.; Schroeder, T.; Falta, J.; Wollschläger, J.; Bäumer, M. Photoemission study of praseodymia in its highest oxidation state: The necessity of in situ plasma treatment J. Chem. Phys. 2011, 134, 054701.

${ }^{29}$ Gevers, S.; Weisemoeller, T.; Schaefer, A.; Zielasek, V.; Bäumer, M.; Wollschläger, J. Structure of oxygen plasma-treated ultra-thin praseodymia films on $\mathrm{Si}(111)$. Phys. Rev. B 2011, 83, 193408.

${ }^{30}$ Schroeder, T.; Lee, T.-L.; Libralesso, L.; Joumard, I.; Zegenhagen, J.; Zaumseil, P.; Wenger, C.; Lupina, G.; Lippert, G.; Dabrowski, J.; Müssig, H.-J. Structure and strain relaxation mechanisms of ultrathin epitaxial $\mathrm{Pr}_{2} \underline{\mathrm{O}}_{3}$ films on $\mathrm{Si}(111)$. J. Appl. Phys. 2005, 97, 074906 (2005).

${ }^{31}$ Weisemoeller, T.; Bertram, F.; Gevers, S.; Greuling, A.; Deiter, C.; Tobergte, H.; Neumann, M.; Giussani, A.; Schroeder, T.; Wollschläger, J. Post deposition annealing induced transition from hexagonal $\mathrm{Pr}_{2} \underline{\mathrm{O}}_{3}$ to cubic $\mathrm{PrO}_{2}$ films on $\mathrm{Si}(111)$. J. Appl. Phys. 2009, 105, 124108 .

${ }^{32}$ Gehl, B.; Leist, U.; Aleksandrovic, V.; Nickut, P.; Zielasek, V.; Weller, H.; Al-Shamery, 
K.; Bäumer, M. Design of a UHV-compatible rf plasma source and its application to self-assembled layers of $\mathrm{CoPt}_{3}$ nanoparticles. Rev. Sci. Instrum. 2006, 77, 083902.

${ }^{33}$ Gevers, S.; Weisemoeller, T.; Bruns, D.; Giussani, A.; Schroeder, T.; Wollschläger, J. Post deposition annealing of praseodymia films on $\mathrm{Si}(111)$ at low temperatures. J. Phys.: Condens. Matter 2011, 23, 115904.

${ }^{34}$ Haire, R. G.; Eyring, L. Handbook on the Physics and Chemistry of Rare Earths, Vol 18., Elsevier Science Ltd, Amsterdam, 1994.

${ }^{35}$ Zaumseil, P.; Schroeder, T. About the strain state of different metal oxide layers epitaxially grown on Si(111). J. Phys. D: Appl. Phys. 2011, 44055403.

${ }^{36}$ Turcotte, R.P.; Warmkessel, J.M.; Tilley, R.J.D.; Eyring, L. On the Phase Interval PrO ${ }_{1.50}$ to $\mathrm{PrO}_{1.71}$ in the Praseodymium Oxide-Oxygen System. J. Solid State Chem. 1971, 3, 265$\underline{272 .}$ 